\title{
Boundary Outlier Centroid Based Reduced Overlapping Image Segmentation
}

\author{
Deepali Virmani ${ }^{1}$, Nikita Jain ${ }^{2, *}$, Ketan Parikh ${ }^{1}$ and Shefali Upadhyaya ${ }^{1}$ \\ ${ }^{1}$ Department of Computer Science, Bhagwan Parshuram Institute of Technology, New Delhi, India \\ ${ }^{2}$ Bharati Vidyapeeth's College of Engineering, New Delhi, India
}

Received 23 August 2018; Accepted 4 November 2018

\begin{abstract}
This paper proposes a novel approach to image segmentation that integrates the improved K-means algorithm with Otsu Segmentation and Watershed algorithm. The conventional K-means algorithm is widely used for image segmentation because of its ease of use and small computation time, but has certain drawbacks such as strongly affected by outliers and may settle at the local minima, thereby leading to poor scaling. Similarly, the traditional watershed algorithm is widely used but has certain drawbacks such as over segmentation and high sensitivity. So the shortcomings of the conventional K-means algorithm and the hide-bound Watershed algorithm are addressed by integrating them such that better segmenting results are obtained. The proposed BOCRO algorithm utilises the combination of clustering algorithm with initial centroids by Centroid Calculation and Overlapping Reduction with Boundary Outliers and is efficient in terms of complexity and produces accurate results. It can be incorporated with a device to detect and segment images to obtain meaningful information.
\end{abstract}

Keywords: Image segmentation, Improved K-means algorithm, Otsu segmentation, Watershed algorithm, BOCRO algorithm

\section{Introduction}

Image segmentation is one of the most important phases of image processing to extract useful and meaningful information in terms of pixels which helps in easier analysis of the image for its comprehension and description. It refers to the process of partitioning or dividing an image in different segments which are commonly termed as pixels. This is usually used to recognize objects or other relevant information in digital images. With image segmentation, the image is partitioned into pixels by assigning labels such that the pixels with same labels exhibit similar characteristics. The pixels in a specific region have similar property. The segments of the image collectively cover the entire image. As per ("Image Segmentation," n.d.), image segmentation is a widely used technique and finds its application in different domains such as image retrieval, medical imaging, various detections such as object detection, face detection, pedestrian detection and break light detection and various recognitions like face recognition, iris recognition and fingerprint recognition. Other applications include traffic control system and video surveillance. So in order to perform image segmentation a variety of methods must be applied to perform the desired task. Various other image segmentation techniques are discussed by Pal and Pal [1] which summarize that neural network models are good for image segmentation in terms of pattern recognition. A survey is conducted by Freixenet et al. [2] which focus on various image segmentation approaches and analyses the usage of region based segmentation and boundary based segmentation.

\footnotetext{
*E-mail address: nikitajain1210@gmail.com

ISSN: 1791-2377 @ 2018 Eastern Macedonia and Thrace Institute of Technology. All rights reserved. doi:10.25103/iestr.115.01
}

With reference to "MathWorks"[3], it can be seen that there are various methods used for performing image segmentation with different techniques. Thresholding method is the simplest method for image segmentation which converts the grey-scale image into binary form consisting of only black and white components. It replaces each pixel in the image with a black pixel if the intensity of the image is less than some predefined constant or a white pixel if the intensity of the image is greater than that constant. One of the thresholding methods is the Otsu's method for image segmentation. Next, colour based segmentation is another technique which incorporates the clustering methods based on colours determining the number of clusters from the number of colours. One of the colour based segmentation method is K-means clustering algorithm. Lastly, there are transform methods for image segmentation which can be applied to an image to convert it from one domain to another which enables easy feature extraction and detection. One of the transform methods used for image segmentation is the Watershed algorithm which is also popularly used.

This paper proposes a novel approach to image segmentation by incorporating various techniques in order to better analyse and obtain meaningful and hidden information from images. It can be applied on different types of images and easily interpreted for further processing thus attaining a better approach to segmenting images for various purposes.

\section{Related Work}

To perform image segmentation various methods are used. In this section, a few of the image segmentation methods such as thresholding based Otsu's method, K-means clustering algorithm, Watershed algorithm, their advantages 
and drawbacks are discussed. This will help in analysing all the popularly used methods to bring out the proposed image segmentation technique (BOCRO) which uses a combination of these techniques to achieve the desired results.

\section{Otsu's Method}

Otsu's method for image segmentation is one of the thresholding based methods for computer vision and image processing which creates binary image from the original grey-scale image by choosing two colours, i.e. black and white. The incorporation of black and white colours is based on some thresholding level such that the image can be segmented easily. It is one of the easiest image segmenting techniques. The algorithm assumes that the image contains two classes of pixels, i.e., foreground pixels and background pixels, it then calculates the optimal threshold separating the two classes so that their collective spread is least. In Otsu's method, the threshold is searched for carefully that minimizes the intra-class, demarcated as a weighted sum of variances of the two classes:

$$
\sigma_{w}^{2}(t)=w_{0}(t) \sigma_{0}^{2}(t)+w_{1}(t) \sigma_{1}^{2}(t)
$$

Weights $w_{0}$ and $w_{1}$ are the probabilities of the two classes separated by a threshold $\mathrm{t}$, and $\sigma_{0}^{2}$ and $\sigma_{1}^{2}$ are variances of these two classes.

The class probability $w_{0,1}(t)$ is computed from the $\mathrm{L}$ bins of the histogram:

$$
\begin{aligned}
& w_{0}(t)=\sum_{i=0}^{t-1} p(i) \\
& w_{1}(t)=\sum_{i=t}^{L-1} p(i)
\end{aligned}
$$

According to Zhang and $\mathrm{Hu}$ [4], two-dimensional Otsu's method behaves well in segmenting images of low SNR than one-dimensional (1D) which helps in overcoming the problem of object size. A comparative analysis of implementing this method has been depicted by Vala and Baxi[5].

\section{K-means Clustering Algorithm}

From the facts stated by Jain[6], it can be observed that the $\mathrm{K}$-means clustering is an unsupervised learning algorithm based on clustering technique. It partitions the data into a fixed class $\mathrm{K}$ on the basis of lessening the squared error function. It is a partitioning algorithm of the feature space clustering algorithms, which is widely used in image segmentation as well. It comes under colour based segmentation category of image segmentation. The steps of $\mathrm{K}$-means clustering algorithm for image segmentation are as depicted in Figure 1.

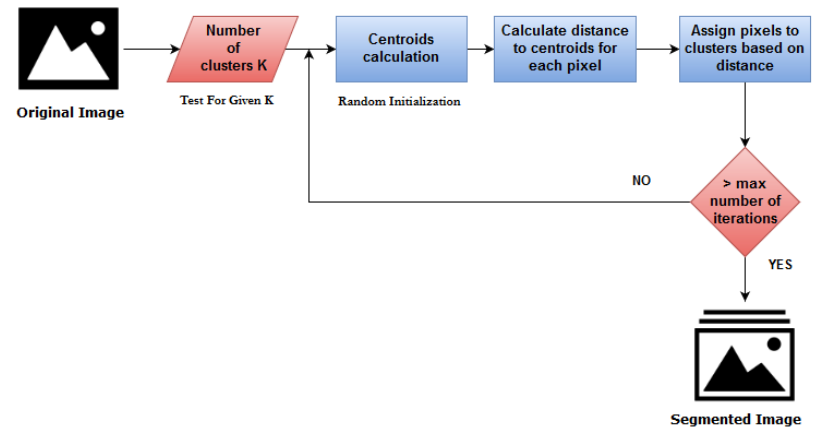

Fig. 1. K-means image segmentation

In this, the number of clusters, $\mathrm{K}$ for the original image is chosen for which random initialization of centroids takes place. Then, for each pixel, distance to centroids is calculated. Later on, pixels are assigned to clusters based on the calculated distances for maximum number of iterations giving output as segmented image.

\section{Watershed Algorithm}

Watershed is the transformation method in image segmentation defined on grey-scale image. As stated by Gauch[7] in graphs, watershed lines may be demarcated on the nodes, on the edges, or hybrid lines on both nodes and edges. Watershed algorithm finds the "watershed ridge lines" and "catchment basins" for the topographical representation of the image to be segmented by treating it as a surface where light pixels represent high elevations and dark pixels represent low elevations. As mentioned by Roerdink and Meijster[8], the pathways of pixels, which congregate in the course of a common minimum, create a catchment basin. Watersheds are the elevated areas that divide the different catchment basins. The partitions, which we aim to obtain, are the catchment basins, and the boundaries between the partitions are the watersheds as shown in Figure 2. Many different algorithms compute watershed.

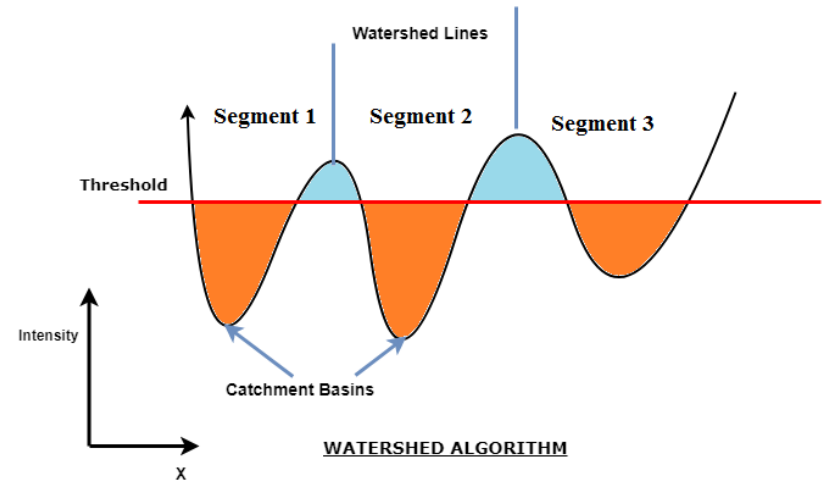

Fig. 2. Watershed ridge lines and catchment basins

Steps followed in Watershed transformation algorithm are as listed below:

- $\quad$ Let $\mathrm{I}$ be the input image and $\mathrm{L}$ is a labelled matrix containing positive integers to the locations of each catchment basin.

$$
\mathrm{L}=\text { watershed(I) }
$$

- Then, the distance transform is calculated for L. It is the distance from every pixel to the nearest nonzero valued pixel.

Distance_Transform(L)

- Use zero-valued elements of L, which are located along the watershed lines, to separate the objects in the original image

$$
\text { image }(\mathrm{L}==0)=0
$$

Table 1 summarizes the three methods of image segmentation in terms of method used, their advantages and limitations giving a generalized view of these methods, thus leading to the proposed BOCRO approach for segmenting the image.

\section{Proposed Boundary Outlier Centroid Based Reduced Overlapping (Bocro) Algorithm}

The proposed BOCRO algorithm is combined on various notations to simulate the procedure of segmentation. The 
uncertainty in determination of number of clusters for $\mathrm{K}$ means segmentation of image results in formation of inaccurate number of clusters followed by initial centroids of the image to be segmented. Initially, random pixels are considered as initial centroids which lead to increased time complexity. Ana chronically, K-means in image segmentation has a major drawback in terms of overlapping of clusters which is reduced by watershed algorithm with morphological operations and various boundary extraction techniques.

The framework for the proposed BOCRO algorithm is shown in Figure 3.

Table 1. Summary of different image segmentation techniques

\begin{tabular}{|c|c|c|c|c|}
\hline & Method & $\begin{array}{l}\text { Mathematical } \\
\text { Representation }\end{array}$ & Advantage & $\begin{array}{l}\text { Limitatio } \\
\mathrm{n}\end{array}$ \\
\hline $\begin{array}{l}\text { Otsu's } \\
\text { Method }\end{array}$ & $\begin{array}{l}\text { Thresholding } \\
\text {-based }\end{array}$ & $\begin{aligned} & f(x, y) \\
= & 1, \text { if } h(x, y) \\
\geq & T \\
= & 0 \text { otherwise }\end{aligned}$ & $\begin{array}{l}\text { Simple; } \\
\text { Quickly } \\
\text { implement } \\
\text { ed }\end{array}$ & $\begin{array}{l}\text { Variances } \\
\text { in the } \\
\text { object } \\
\text { areas and } \\
\text { the } \\
\text { backgroun } \\
\text { d } \\
\text { intensities } \\
\text { produce } \\
\text { noise } \\
\text { distorting } \\
\text { the image } \\
\text { as per } \\
\text { Otsu's } \\
\text { Method[1 } \\
\text { 5]; } \\
\text { Small } \\
\text { object size } \\
\text { and } \\
\text { considerati } \\
\text { on of only } \\
\text { two } \\
\text { classes }\end{array}$ \\
\hline $\begin{array}{l}\text { K-means } \\
\text { Segmentati } \\
\text { on }\end{array}$ & Colour-based & $\underset{-\mu_{j} \|}{\arg \min } \sum_{i=1}^{k} \sum_{j=1}^{k} \| x_{i}$ & $\begin{array}{l}\text { Less } \\
\text { number of } \\
\text { clusters- } \\
\text { faster } \\
\text { computatio } \\
\text { nal time }\end{array}$ & $\begin{array}{l}\text { Number of } \\
\text { clusters } \\
\text { cannot be } \\
\text { pre- } \\
\text { determine } \\
\text { d; Random } \\
\text { initializati } \\
\text { on of } \\
\text { cluster } \\
\text { makes it } \\
\text { less } \\
\text { efficient as } \\
\text { observed } \\
\text { by } \\
\text { Chaturved } \\
\text { i and } \\
\text { Rajavat[14 } \\
\text { ]; Strongly } \\
\text { affected } \\
\text { by } \\
\text { outliers; } \\
\text { May settle } \\
\text { at local } \\
\text { minima }\end{array}$ \\
\hline $\begin{array}{l}\text { Watershed } \\
\text { Algorithm }\end{array}$ & $\begin{array}{l}\text { Transformati } \\
\text { on-based }\end{array}$ & $\begin{array}{l}T_{f}^{P}(p, q) \\
=\sum_{i=0}^{l(P)-1} \operatorname{cost}_{f}\left(p_{i}, p_{i} .\right.\end{array}$ & $\begin{array}{l}\text { Extensivel } \\
\text { y segments } \\
\text { image with } \\
\text { very clear } \\
\text { demarcatio } \\
\text { ns }\end{array}$ & $\begin{array}{l}\text { Over- } \\
\text { segmentati } \\
\text { on and } \\
\text { high } \\
\text { sensitivity }\end{array}$ \\
\hline
\end{tabular}

The framework depicts uploading the image, converting to grayscale, obtaining the histogram in order to find peaks, simultaneously applying Cluster Identification and Centroid Calculation to obtain the threshold value for calculation of initial centroids to be passed in the improved clustering algorithm. After clustering, Overlapping Reduction is applied that creates watershed ridge lines and catchment basins. This helps in obtaining Boundary Outliers for each and every line and basin in order to generate the final segmented image for the original image.

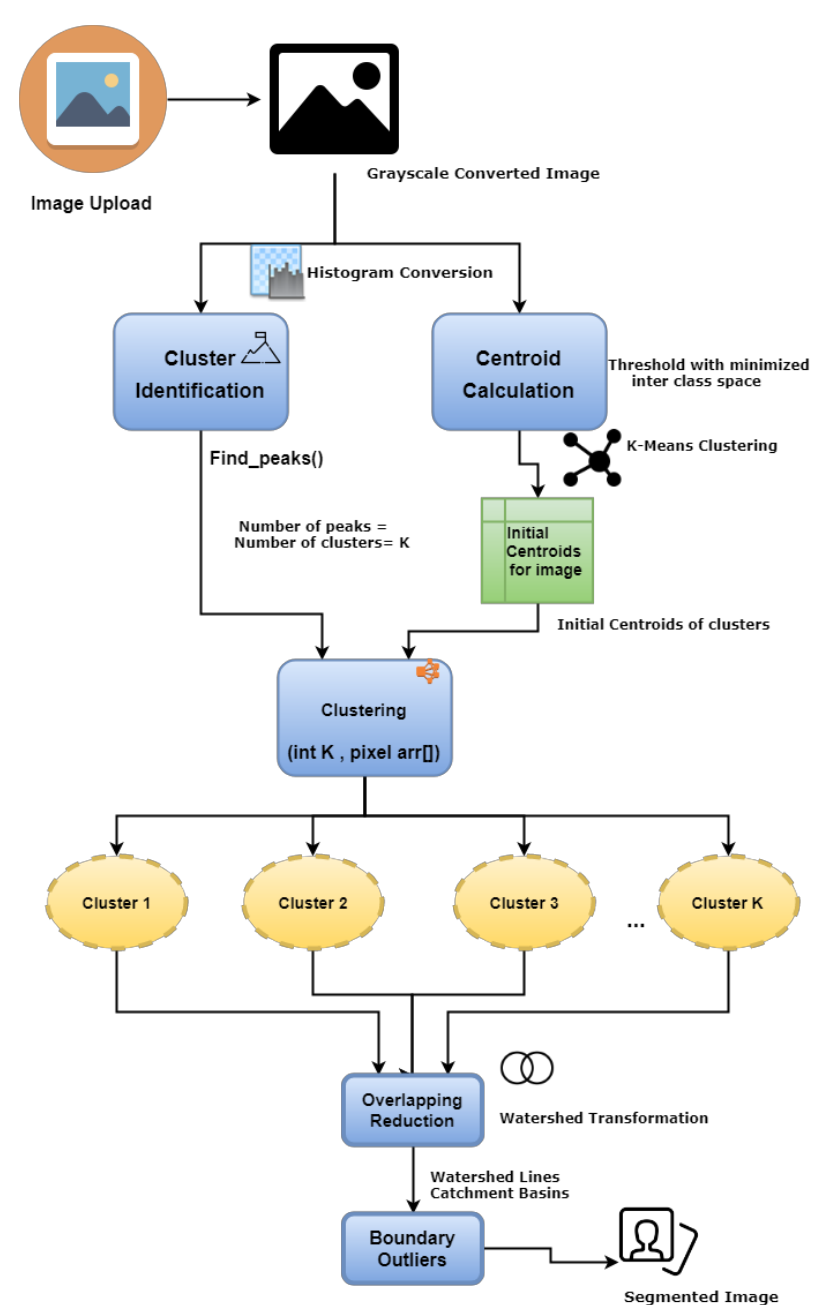

Fig. 3. Framework for the proposed BOCRO algorithm

\section{Finding Number of Clusters (Cluster Identification)}

The proposed BOCRO algorithm first converts image to grayscale. After grey-scaling, histograms are obtained and peaks are calculated for the grey-scaled image. The number of peaks is used to determine the number of clusters for the $\mathrm{K}$-means clustering algorithm which is proposed by Virmani et al.[9].

Firstly, the histogram is transformed and then the number of peaks is calculated based on some threshold value where the peak corresponds to degree of intensity of a pixel. This threshold value is used as peak point. If the point neighbouring to the peak point is less than the threshold for the calculated distance it will be rejected, and finally the peak point will be marked on the histogram to calculate the peak number which determines the number of clusters. Following are the algorithmic steps for cluster identification of the image to be segmented:

3.1.1 Cluster_Identification (Image I, Threshold $\mathrm{Th}_{\text {peaks }}$ )
3.1.11 Pixels $\mathrm{p}[] \leftarrow$ I.toPixels $($ )
3.1.12 Hashmap $<$ Pixel,Int $>$ histogram
3.1.13 integer array peaks[], dis[]
3.1.14 for $\mathrm{i} \leftarrow 0$ to p.length // Evaluating intensity
per pixel $\begin{aligned} & \mathrm{j} \leftarrow 0 \\ & \text { if }(\text { histogram.exists }(\mathrm{p}[\mathrm{i}])) \text { then }\end{aligned}$




value $\leftarrow$ histogram.getValue ()
increment value
remove $\mathrm{p}[\mathrm{i}]$ from histogram
histogram.add(p[i], value)

The results section depicts the labelled diagram of peaks on histogram which are used to determine the number of clusters for K-means segmentation.

\section{Centroid Calculation}

To find out the correct threshold level, Otsu segmentation is used by the proposed BOCRO algorithm by dividing the image into two modes, i.e. black and white. Centroid calculation uses binary image to calculate the threshold value, i.e. $\mathrm{Th}_{\text {centroid }}$ correctly. Following are the algorithmic steps for finding the correct threshold for the image after cluster identification:

Let $\omega_{\mathrm{i}}$ be the probability of $\mathrm{i}^{\text {th }}$ class and $\mu_{\mathrm{i}}$ be the $\mathrm{i}^{\text {th }}$ class mean,

where i denotes the number of clusters and Initial_centroids [] be the array of centres

\subsubsection{Centroid_Calculation(Histogram h, Probability pb[] ) 3.2.1 Initialize $\mathrm{w}_{\mathrm{i}}$ and $\mu_{\mathrm{i}}$}

$\mu_{T}=\sum_{i=0}^{L-1} i p(i)$

3.2.12 For Threshold $t \leftarrow 1$ to maximum intensity

$w_{0}(t)=\sum_{i=0}^{t-1} p(i)$

$w_{1}(t)=\sum_{i=t}^{L-1} p(i)$

$\mu_{0}(t)=\sum_{i=0}^{t-1} i \frac{p(i)}{w_{0}}$
$\mu_{1}(t)=\sum_{i=t}^{L-1} i \frac{p(i)}{w_{1}}$

$$
{\sigma_{b}}^{2}(t)=\sum_{i=0}^{i} \omega_{i}(t)\left(\mu_{i}(t)-\mu_{T}\right)^{2}
$$

End loop

3.2.13 Desired Threshold $\operatorname{Th}_{\text {centroid }} \leftarrow \operatorname{Max}\left(\sigma_{b}{ }^{2}(t)\right)$ Th $\left.h_{\text {centroid }}\right)$

3.2.14 Cluster $\mathrm{c}[] \leftarrow \mathrm{K}$-means(Desired_Threshold

3.2.15 For $\mathrm{i} \leftarrow 0$ to c.length Initial_centroids[i] mean(c[i].pixels) // mean of data points End loop

3.2.16 Return Initial_centroids

Thus the desired threshold $\mathrm{Th}_{\text {centroid }}$ as obtained from the algorithm helps in determining the classes of clusters such that initial centroids could be provided in the Cluster Identification, thereby reducing the overhead to calculate initial centres.

\section{Overlapping Reduction}

After finding the initial centroids, the obtained binary image is inverted to calculate the distance transform. The distance transform, i.e. Distance_Transform() in Watershed algorithm as given by Meijster and Roerdink[10] calculates the distance from every pixel to the nearest non-zero valued pixel. The image is modified such that the background pixels and the extended maxima pixels are forced to be the only local minima in the image. As mentioned by $\mathrm{Ng}$ et al. [11], watershed algorithm is able to provide a complete splitting up of the image in separated regions even if the contrast of the image is poor. Also, it helps in reducing the cluster overlapping by clear demarcation with ridge lines. Following are the algorithmic steps for overlapping reduction for the image after centroid calculation:

Let I be the input clustered image applied with Otsu segmentation and $\mathrm{L}$ is a labelled matrix containing positive integers to the locations of each catchment basin.

3.3.1 Overlapping_Reduction(Image clustered_image)

3.3.11 I $\leftarrow$ imread(clustered image) //Reading image 3.3.12 L $\leftarrow$ watershed(I , Clusters $\mathrm{c}[\mathrm{]}) / /$ Reducing clusters overlapping

3.3.13 L $\leftarrow$ Distance_Transform $(\mathrm{L})$

3.3.14 Ridge_Linesr[] $\leftarrow$ image $(\mathrm{L}==0)=0$

3.3.15 For $\mathrm{i} \leftarrow 0$ to r.length

Divide segments into two basins

End loop

3.3.16 Obtain clusters with reduced overlapping with ridge lines

3.3.17 return image

Use zero-valued elements of $\mathrm{L}$, which are located along the watershed lines, to separate the objects in the original image.

\section{Boundary Outliers}

After Overlapping Reduction on the image, the boundaries are extracted in order to clearly demarcate the segmentation of image. As observed by Arbelaez [12], different methods are followed to segment the image with extraction of boundaries. For boundary outliers, a simple linear iterative clustering (SLIC) algorithm is used. This algorithm groups pixels into regions with similar values. Using these regions 
in image segmentation reduces the complexity. Further, the region boundaries are computed in the form of mask on the image. Thus, the final segmented image is obtained. Following are the algorithmic steps for boundary outlier determination to obtain the final segmented image:

\subsubsection{Boundary_Outliers(Image image)}

3.4.11 I' $\leftarrow$ super_pixels(image) // simple linear iterative clustering

3.4.12 region_image $\leftarrow$ boundary_masks(I')

3.4.13 return region image

\section{BOCRO Algorithm}

The following algorithmic steps explain the complete working of the proposed BOCRO method:

\begin{tabular}{|l|}
\hline 3.5.1 I $\leftarrow$ read_image(address) \\
3.5.2 Ig $\leftarrow$ GrayScale $(\mathrm{I})$ \\
3.5.3 Histogram $\mathrm{h} \leftarrow \mathrm{ImHist}(\mathrm{Ig})$ \\
\hline
\end{tabular}

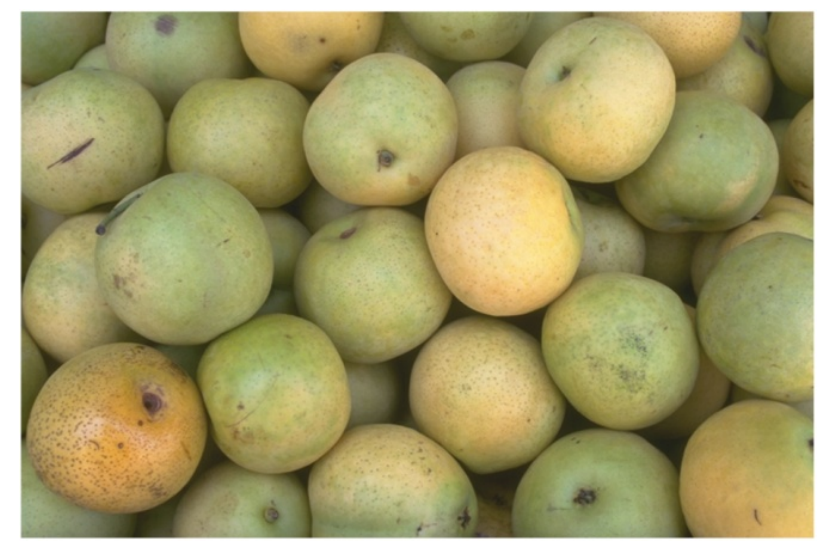

Fig. 4. Pears image

(a) Original image

The grayscale pears image is used to create a histogram which helps in calculating the peaks in terms of pixel intensity which is used to find the number of clusters in terms of number of peaks. The histogram is depicted in Figure 5.

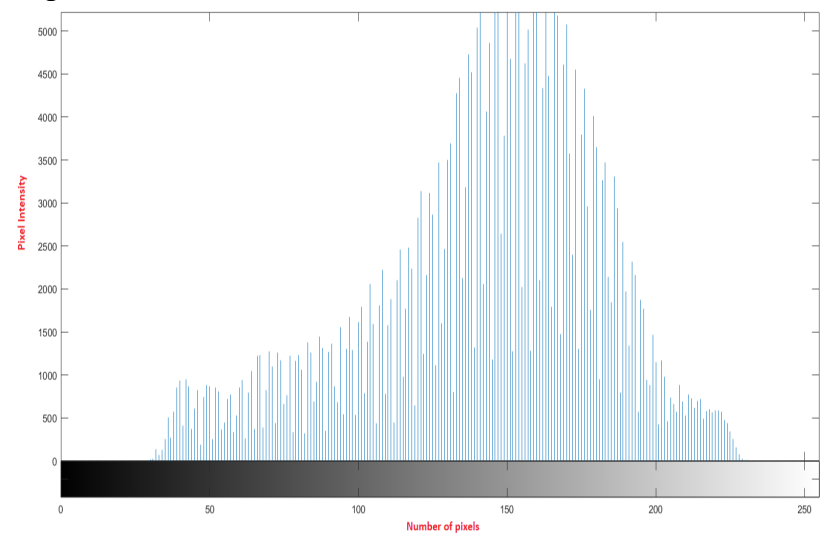

Fig. 5. Histogram showing number of pixels and their intensity in the grayscale pears image

The peaks in the above histogram are obtained such that local maximum intensity could be found as shown in Figure 6 and these peaks are used as number of clusters in the further processing of the image by Cluster Identification. The number of peaks is found to be 5 from the obtained histogram.

\begin{tabular}{|l|}
\hline 3.5.4 Number of clusters \\
$\mathrm{K} \leftarrow$ Cluster_Identification $\left(I g, T h_{\text {peaks }}\right)$ \\
3.5.5 Centroids c[] $\leftarrow$ Centroid_Calculation(Histogram $\mathrm{h})$ \\
3.5.6 Clusters[] $\leftarrow$ K-means $(\mathrm{K}, \mathrm{c}[])$ \\
3.5.7 $\mathrm{I}_{\text {seg }} \leftarrow$ Overlapping_Reduction(Clusters[].to image ()$)$ \\
3.5.8 $\mathrm{I}_{\text {seg }}, \leftarrow$ Boundary_Outliers $\left(\mathrm{I}_{\text {seg }}\right)$ \\
Return $\mathrm{I}_{\text {seg }} / / /$ Segmented Image \\
\hline
\end{tabular}

\section{Results and Analysis}

The results were observed for the image corresponding to pears having very minute distinguishable colours. It is used in step by step form for various transformations. Figure 4(a) depicts the original pears image. In the next step, the pears image is transformed to grayscale pears image as shown in Figure 4(b).

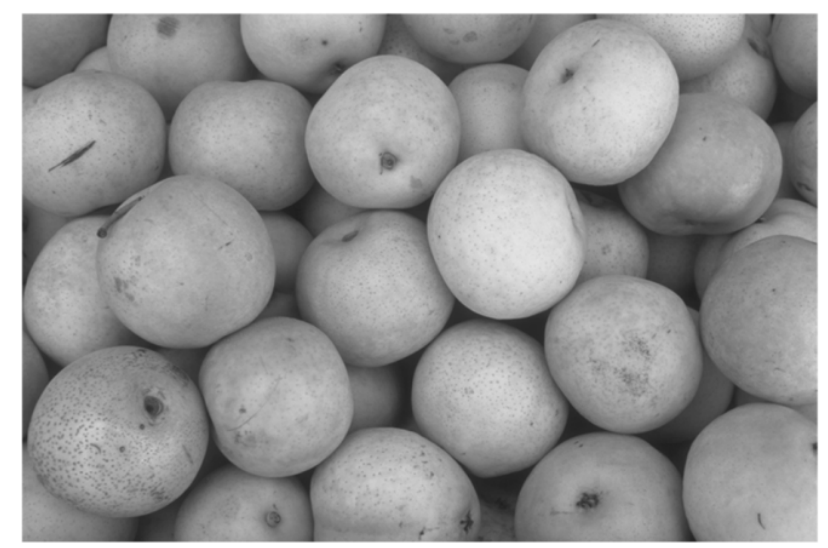

(b) Grayscale image

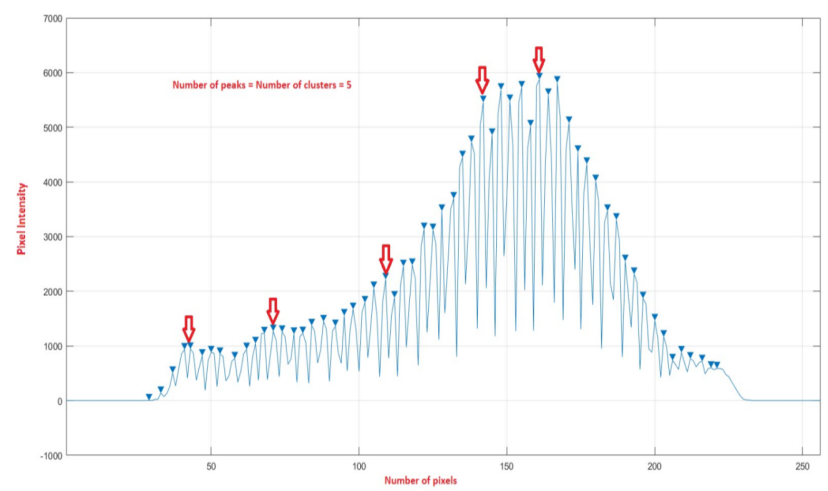

Fig. 6. Histogram showing peaks marked which is used to obtain number of clusters

Now, Cluster Identification of the proposed BOCRO algorithm is applied on the grayscale image such that the number of clusters determines the number of colours in the image. Thus, the image is segmented on the basis of colours as shown in Figure 7(a) with $\mathrm{K}=3$ and Figure 7(b) with $\mathrm{K}=5$.

The 3 clusters obtained are shown individually in the following Figure 8 .

The 5 clusters obtained are shown individually in the following Figure 9. There are 5 colours which identify the 5 clusters for the single image. As can be seen from the obtained clusters, all the colours are different shades of green, thus better clustering is observed. Thus, the number of peaks correctly determined the number of clusters. 
Also, for orl face dataset image the number of peaks correctly determines the number of clusters for the image,

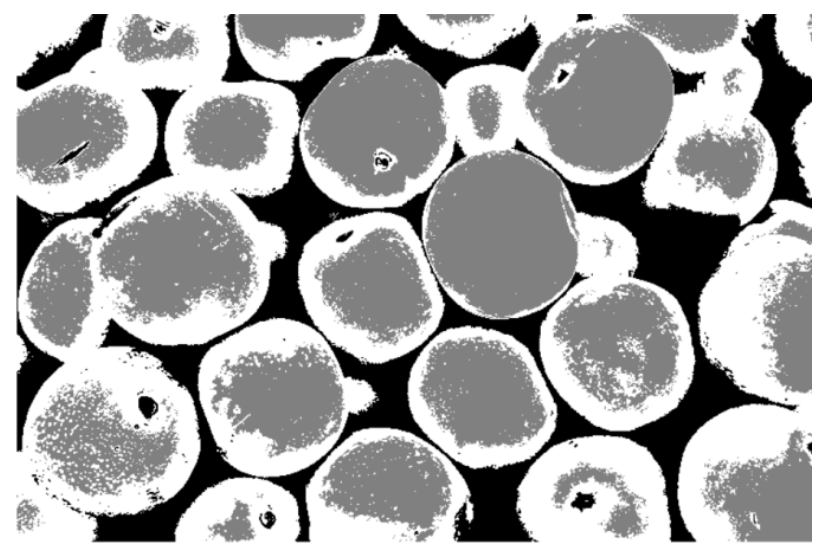

(a) $\mathrm{K}=3$

Fig. 7. Cluster Identification based segmentation of pears image with 3 and 5 clusters

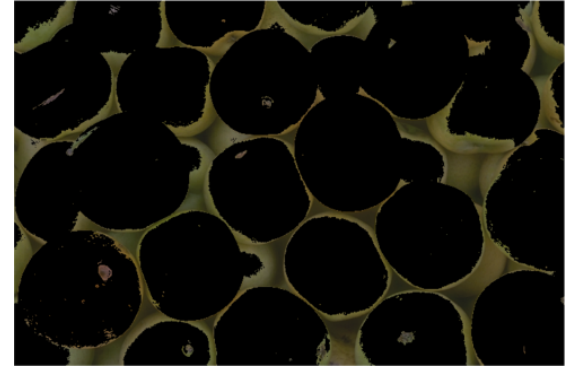

(a) Objects in cluster 1

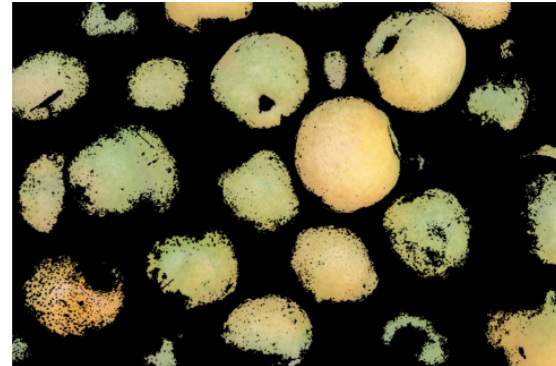

(b) Objects in cluster 2

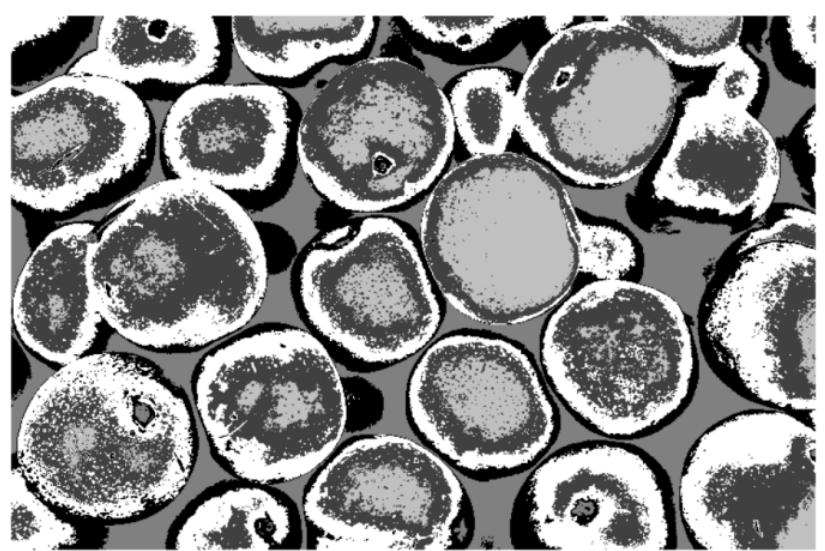

(b) $\mathrm{K}=5$

Fig. 8. Coloured clusters obtained from Cluster Identification for $\mathrm{K}=3$ for pears image

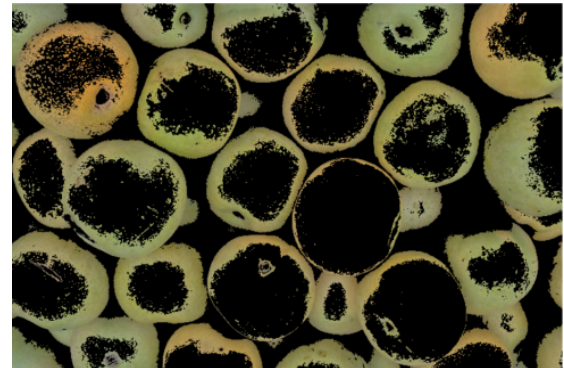

(c) Objects in cluster 3
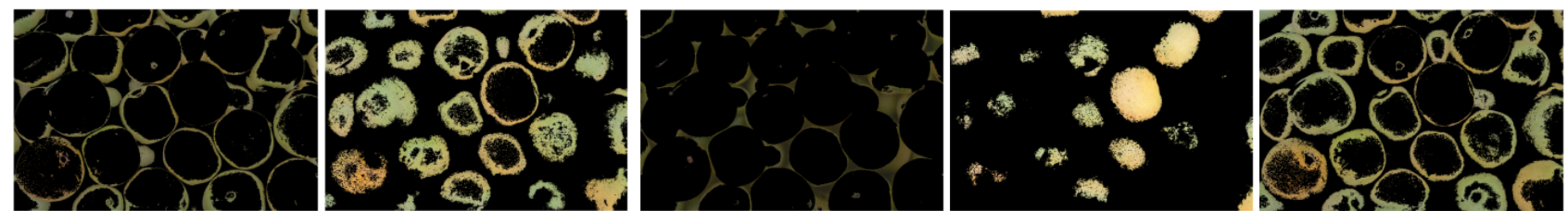

(a)Cluster 1 objects (b)Cluster 2 objects (c)Cluster 3 object (d)Cluster 4 objects (e)Cluster 5 objects

Fig. 9. Coloured clusters obtained from Cluster Identification for $\mathrm{K}=5$ for pears image
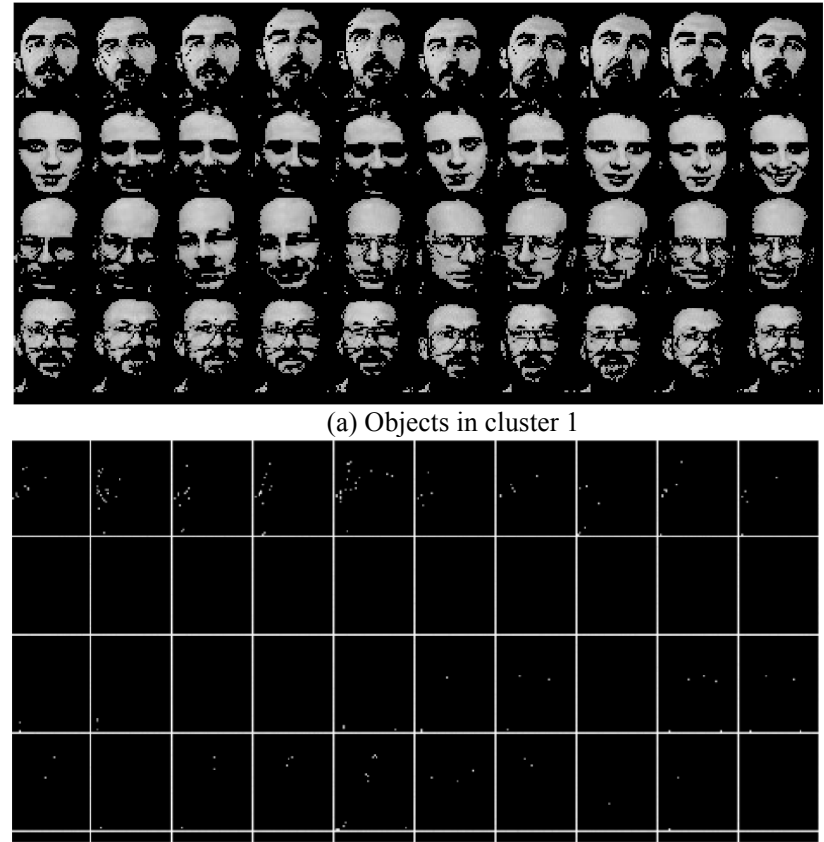

(c) Objects in cluster 3

Fig. 10. Clusters obtained from Cluster Identification for $\mathrm{K}=4$ for orl face dataset

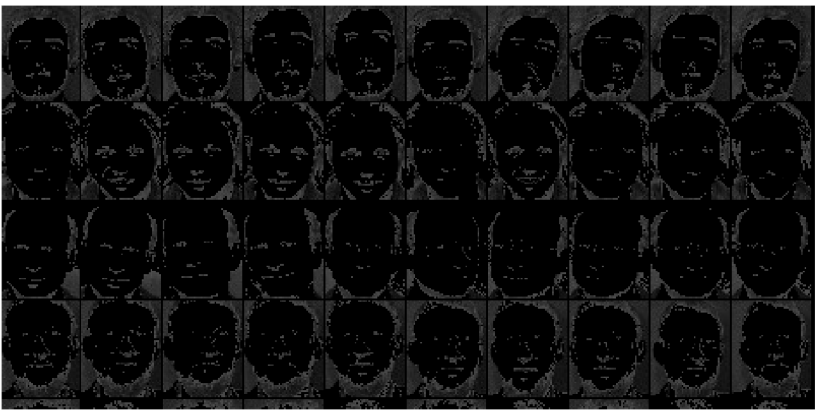

(b) Objects in cluster 2

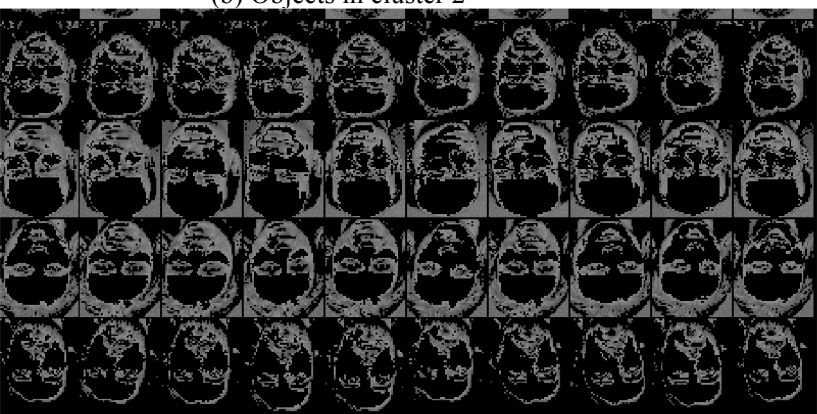

(d) Objects in cluster 4 
The image containing 5 clusters of pears image is used for further processing as it best describes the clusters in terms of number of peaks as well. The contrast of the image is adjusted so that all the clusters are clearly defined and separated as shown in Figure 11.

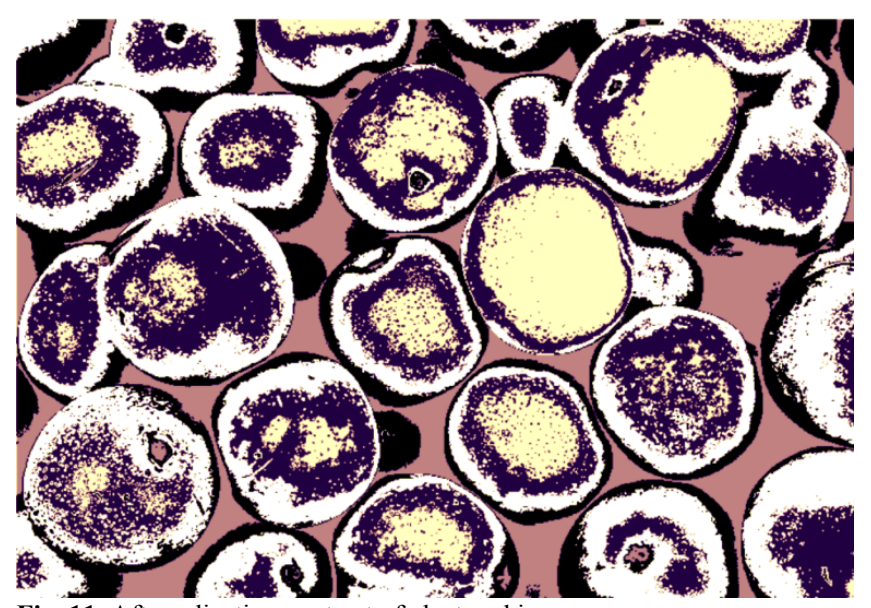

Fig. 11. After adjusting contrast of clustered image

Now, Otsu's Method is applied on this image for calculating the correct threshold. The resulting image segmentation after centroid calculation is a binary image as depicted by Figure 12, which helps to improve clustering by providing initial cluster centroids.

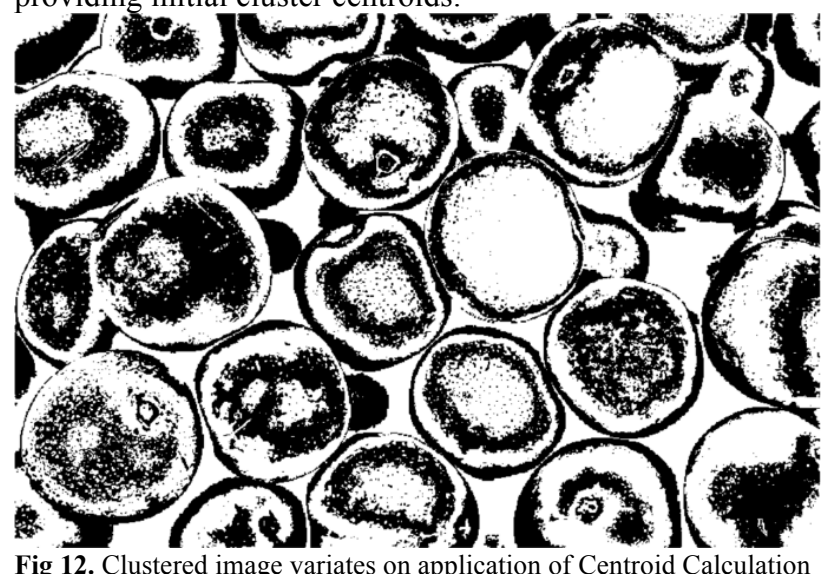

The colours of binary image are inverted to apply Overlapping Reduction of the proposed BOCRO algorithm as depicted in Figure 13.

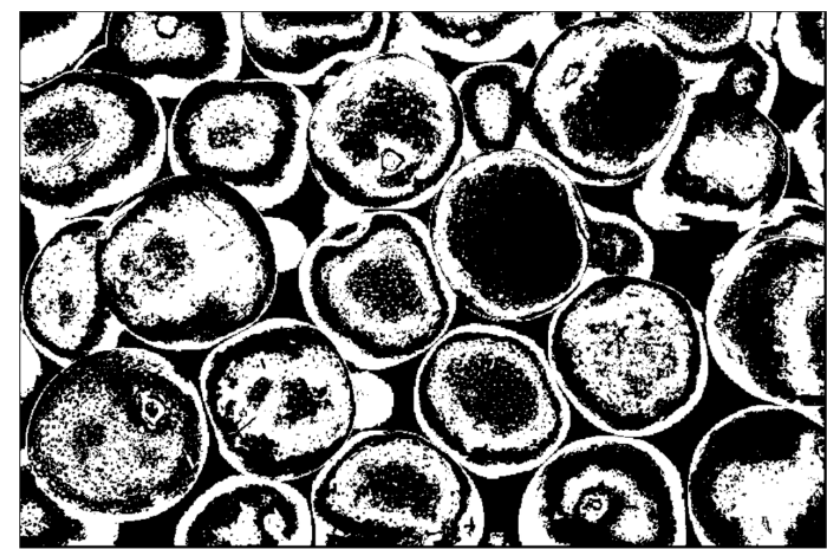

Fig. 13. Inverted binary clustered image
The inverted black and white image is used to calculate the distance transform which is the distance from every pixel to the nearest non-zero valued pixel such that Overlapping Reduction is applied completely. The resulting image is converted back to RGB components in order to obtain complete segmented pears image with ridge lines and catchment basins as shown in Figure 14.

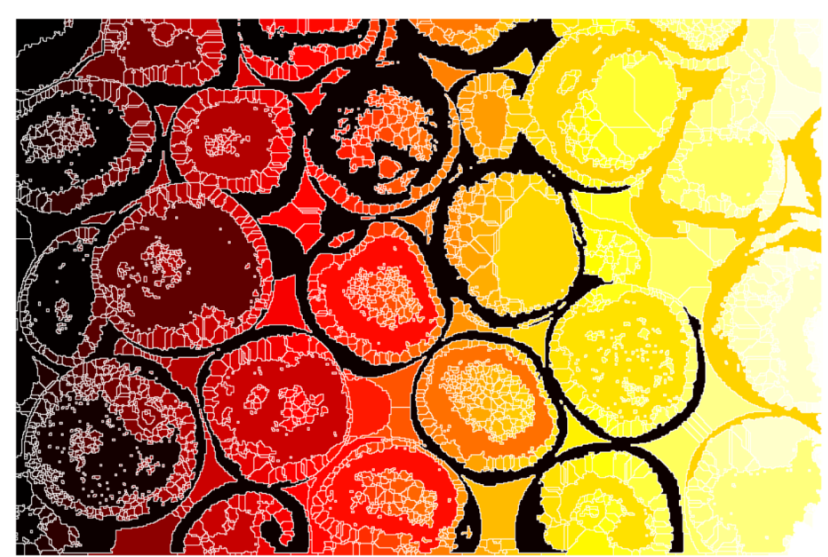

Fig. 14. Complete segmented image with ridges and basins after Overlapping Reduction

Further, the ridge lines are directly mapped on the original clustered image as depicted in Figure 15. These lines clearly depict the in-depth bifurcations needed for the image to be segmented, thus improving the quality of image segmentation.

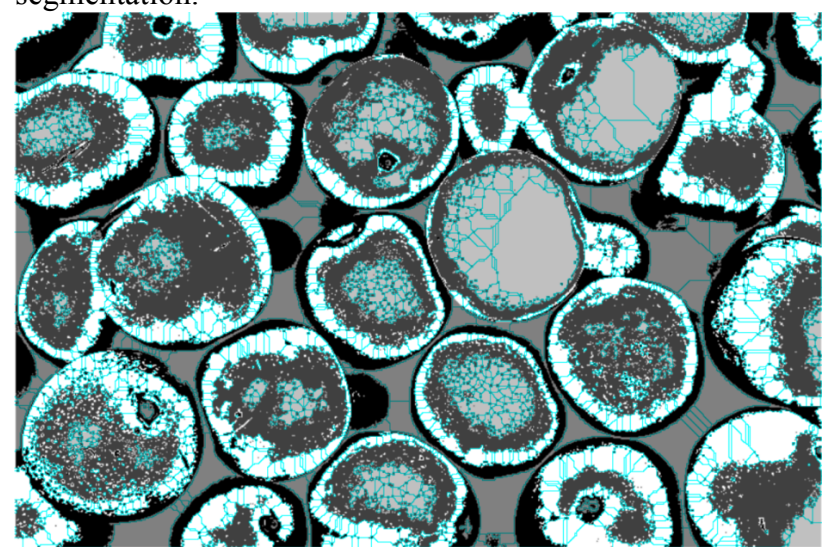

Fig. 15. Clustered image with ridge lines for pears image

Also, for orl face dataset image, clear demarcations for the final segmented image can be seen as obtained from the proposed BOCRO algorithm in Figure 16.

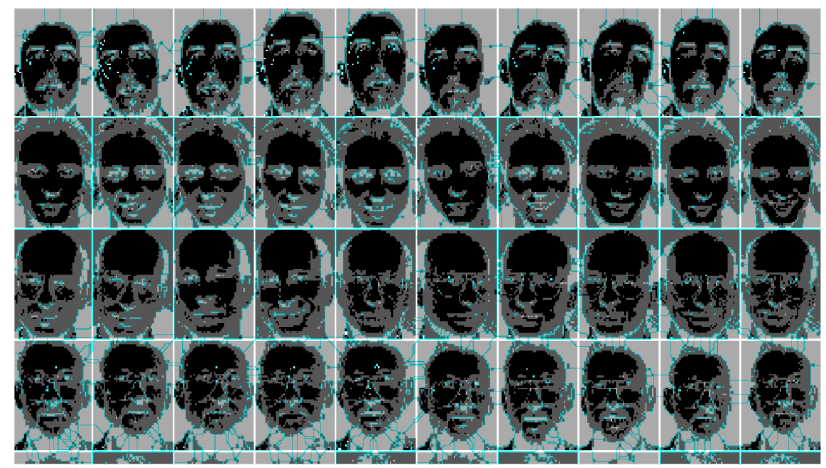

Fig. 16. Clustered image with ridge lines for orl face dataset 
Finally, to display the segmented image more accurately, Boundary Outliers of the proposed BOCRO algorithm is performed on the pears image obtained after Overlapping Reduction. Boundary Outliers image results in better segmentation output as it clearly depicts the required segmented regions for the original pears image. Figure 17(a) and Figure 17(b) clearly depict the difference between the original image boundaries and the final segmented image boundaries from the proposed BOCRO algorithm.

It can be seen that the final segmented image boundaries are much better than the original image boundaries, thereby producing desired image segmentation. Thus, the proposed BOCRO approach for image segmentation produces remarkable improvement in segmenting images.

The following Figure 18 depicts the mapping of the original image boundaries over the segmented image boundaries. It can be clearly seen that the original image has been very precisely segmented by the proposed BOCRO algorithm. The green demarcations represent the original image boundaries while the complete inner boundaries are depicted in the final segmented image.

Similarly, the comparison between the above mentioned images is depicted with the help of a bar graph as shown in Figure 19(a) and Figure 19(b) respectively. The bar graph representation for original image clearly depicts less segmentation as compared to the bar graph representation of the final segmented image by the proposed BOCRO algorithm.

A review on various segmenting techniques is observed by Kaur and Kaur[13]. Table 2 analyses different segmenting techniques and the proposed hybrid segmentation technique.

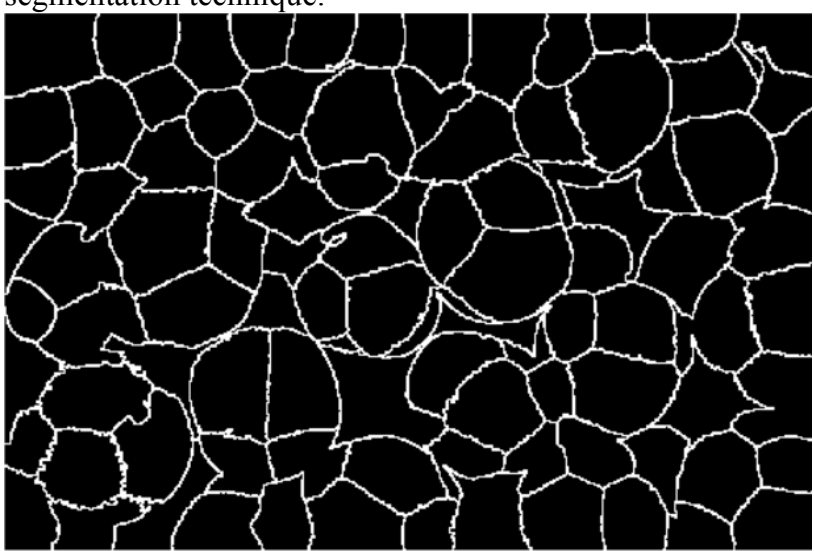

(a) Original boundaries

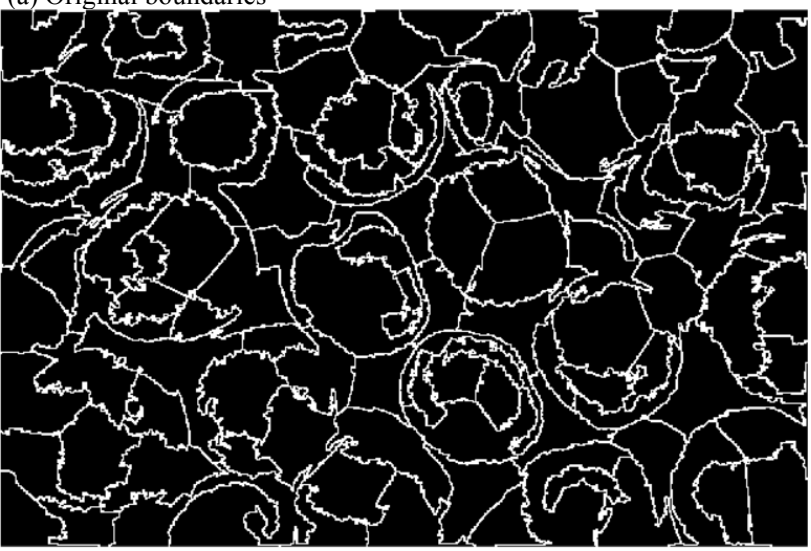

(b) Final segmented boundaries

Fig. 17. Boundary Outliers for pears image

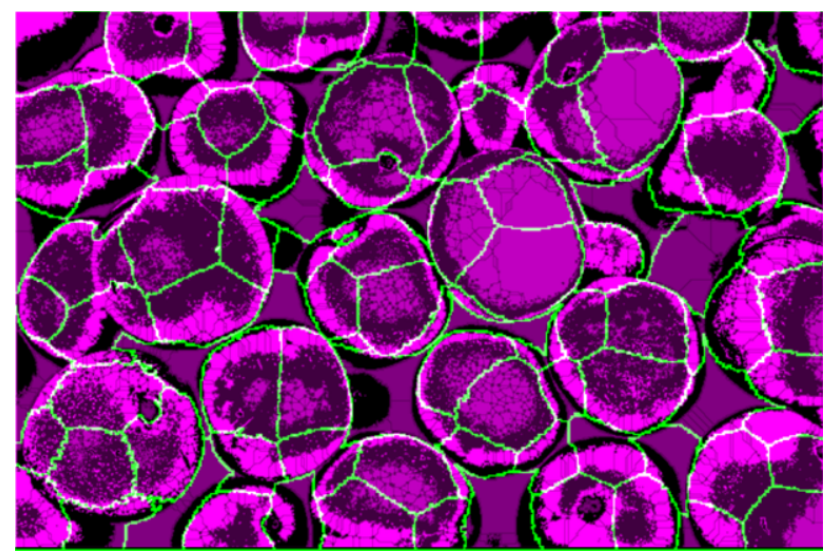

Fig. 18. Comparison of original pears image and segmented image from proposed BOCRO algorithm

Table 2. Comparison of proposed BOCRO algorithm with other segmenting techniques

\begin{tabular}{|c|c|c|}
\hline $\begin{array}{l}\text { Segmentation } \\
\text { Technique }\end{array}$ & Description & Features \\
\hline $\begin{array}{l}\text { Proposed } \\
\text { BOCRO } \\
\text { Method }\end{array}$ & $\begin{array}{l}\text { Hybrid of improved } \\
\text { K-means (clustering), } \\
\text { Threshold and } \\
\text { Watershed } \\
\text { transformation }\end{array}$ & $\begin{array}{l}\text { Predetermines number of } \\
\text { clusters and initial } \\
\text { centroids of clusters; Less } \\
\text { time complexity; Detects } \\
\text { boundaries accurately }\end{array}$ \\
\hline $\begin{array}{l}\text { Thresholding } \\
\text { Method }\end{array}$ & $\begin{array}{l}\text { Based on the } \\
\text { histogram peaks of the } \\
\text { image to find } \\
\text { particular threshold } \\
\text { values }\end{array}$ & $\begin{array}{l}\text { Simplest method; highly } \\
\text { dependent on peaks }\end{array}$ \\
\hline $\begin{array}{l}\text { Colour Based } \\
\text { (Clustering) } \\
\text { Method }\end{array}$ & $\begin{array}{l}\text { Based on division into } \\
\text { homogeneous clusters }\end{array}$ & $\begin{array}{ll}\text { Cannot predict number of } \\
\text { clusters; } & \text { determining } \\
\text { membership } & \text { function is } \\
\text { not easy } & \end{array}$ \\
\hline $\begin{array}{l}\text { Watershed } \\
\text { Method }\end{array}$ & $\begin{array}{l}\text { Based on topological } \\
\text { interpretation }\end{array}$ & $\begin{array}{l}\text { Detected boundaries are } \\
\text { continuous; complex } \\
\text { calculation of gradients }\end{array}$ \\
\hline $\begin{array}{l}\text { Edge \& Region } \\
\text { Based Method }\end{array}$ & $\begin{array}{l}\text { Based on } \\
\text { discontinuity } \\
\text { detection and } \\
\text { partitioning image } \\
\text { into homogeneous } \\
\text { regions }\end{array}$ & $\begin{array}{l}\text { Not suitable for wrong } \\
\text { detected or too many } \\
\text { edges; expensive method } \\
\text { in terms of time and } \\
\text { memory }\end{array}$ \\
\hline
\end{tabular}

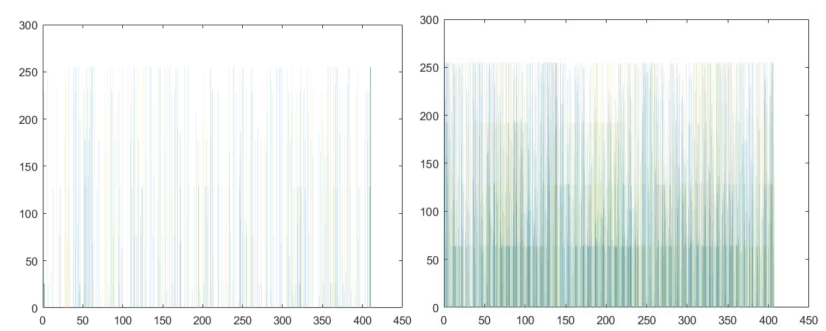

Fig. 19. Comparison of original boundary image and final segmented image in terms of bar graph representation

\section{Conclusion}

Image segmentation is a wide domain where several segmentation algorithms are used in order to produce good results. This paper provides a novel approach to improve Kmeans segmentation algorithm and builds a hybrid with Otsu's method and the Watershed algorithm. The proposed BOCRO algorithm calculates number of required peaks from the grey histogram of the image which determine the number of clusters for K-means segmentation. Further, Otsu segmentation helps in calculating the correct threshold level for the binary image thereby providing initial centroids for the K-means algorithm. Next, watershed algorithm is used to completely segment the image with formation of ridge lines 
and catchment basins thereby reducing overlapping in clusters. Finally, boundary extraction is performed on the final segmented image to clearly display segmentation output of the image. The results clearly depict the differences in original and segmented image boundaries. Hence, it is concluded that the proposed BOCRO algorithm results in improved segmentation outputs as compared with the other segmentation algorithms. The effectiveness of integrating the segmentation algorithms can be seen with the improved and accurate results obtained.

This is an Open Access article distributed under the terms of the Creative Commons Attribution Licence

\section{References}

[1]Pal, N. R., \& Pal, S. K. A review on image segmentation techniques. Pattern recognition, 26(9), 1277-1294, (1993).

[2] Freixenet, J., Muñoz, X., Raba, D., Martí, J., \& Cufí, X,Yet another survey on image segmentation: Region and boundary information integration. In European conference on computer vision (pp. 408422). Springer, Berlin, Heidelberg, (2002).

[3] MathWorks, (2018), The MathWorks Inc., Retrieved February 14, 2018 from https://in.mathworks.com/discovery/imagesegmentation.html

[4] Zhang, J., \& Hu, J. Image segmentation based on 2D Otsu method with histogram analysis. In Computer Science and Software Engineering, 2008 International Conference on (Vol. 6, pp. 105108). IEEE, (2008).

[5] Vala, M. H. J., \& Baxi, A. A review on Otsu image segmentation algorithm. International Journal of Advanced Research in Computer Engineering \& Technology (IJARCET), 2(2), pp-387, (2013).

[6] Jain, A. K.Data clustering: 50 years beyond K-means. Pattern recognition letters, 31(8), 651-666, (2010).

[7] Gauch, J. M, Image segmentation and analysis via multiscale gradient watershed hierarchies. IEEE transactions on image processing, 8(1), 69-79, (1999).

[8]Meijster, A., \& Roerdink, J. B. (1998, September). A disjoint set algorithm for the watershed transform. In Signal Processing Conference (EUSIPCO 1998), 9th European (pp. 1-4). IEEE.
[9] Virmani, D., Jain, N., Parikh, K., Upadhyaya, S., \& Srivastav, A. Proficient Normalised Fuzzy K-Means With Initial Centroids Methodology. International Journal of Knowledge Discovery in Bioinformatics (IJKDB), 8(1), 42-59,(2018).

[10] Roerdink, J. B., \& Meijster, A. The watershed transform: Definitions, algorithms and parallelization strategies. Fundamenta informaticae, 41(1, 2), 187-228,(2000).

[11] Ng, H. P., Ong, S. H., Foong, K. W. C., Goh, P. S., \& Nowinski, W. L. Medical image segmentation using k-means clustering and improved watershed algorithm. In Image Analysis and Interpretation, 2006 IEEE Southwest Symposium on (pp. 61-65). IEEE,(2006).

[12] Arbelaez, P. Boundary extraction in natural images using ultrametric contour maps. In Computer Vision and Pattern Recognition Workshop, 2006. CVPRW'06. Conference on (pp. 182-182). IEEE,(2006).

[13] Kaur, D., \& Kaur, Y. Various image segmentation techniques: a review. International Journal of Computer Science and Mobile Computing, 3(5), 809-814,(2014).

[14] Chaturvedi, E. N., \& Rajavat, E. A. (2013). An improvement in Kmean clustering algorithm using better time and accuracy. Int. J. Program. Lang. Appl, 3, 13-19.

[15] Otsu's Method. (n.d.). In Wikipedia. Retrieved January 19, 2018, from https://en.wikipedia.org/wiki/Otsu's_method 